\title{
Segregation of chain ends to the surface of a polymer melt: effect of surface profile versus chain discreteness
}

\author{
P. Mahmoudi ${ }^{1}$ and M. W. Matsen ${ }^{1,2,3, a}$ \\ 1 Department of Chemical Engineering, University of Waterloo, Waterloo, Ontario, Canada \\ 2 Department of Physics \& Astronomy, University of Waterloo, Waterloo, Ontario, Canada \\ 3 Waterloo Institute for Nanotechnology, University of Waterloo, Waterloo, Ontario, Canada
}

Received: October 30, 2019

\begin{abstract}
Silberberg has argued that the surface of a polymer melt behaves like a reflecting boundary on the random-walk statistics of the polymers. Although this is approximately true, independent studies have shown that violations occur due to the finite width of the surface profile and to the discreteness of the polymer molecule, resulting in an excess of chain ends at the surface and a reduction in surface tension inversely proportional to the chain length, $N$. Using self-consistent field theory (SCFT), we compare the magnitude of these two effects by examining a melt of discrete polymers modeled as $N$ monomers connected by Hookean springs of average length, $a$, next to a polymer surface of width, $\xi$. The effects of the surface width and the chain discreteness are found to be comparable for realistic profiles of $\xi \sim a$. A semi-analytical approximation is developed to help explain the behavior. The relative excess of ends at the surface is dependent on the details of the model, but in general it decreases for shorter polymers. The excess is balanced by a long-range depletion that has a universal shape independent of the molecular details. Furthermore, the approximation predicts that the reduction in surface energy equals one unit of $k_{B} T$ for every extra chain end at the surface.
\end{abstract}

PACS. XX.XX.XX No PACS code given

\section{Introduction}

Polymer melts are not only technologically important, they also represent one of the most fundamental systems in soft condensed matter physics. Their equilibrium properties are of particular interest. Fortunately, the hard-core interactions that would potentially complicate their behavior are screened to a good approximation [1], and consequently the polymers obey random-walk statistics with an average end-to-end length of $a N^{1 / 2}$, where $a$ is the statistical bond length and $N$ is the degree of polymerization. The next issue is how the polymer statistics are affected by a surface, but this too turns out to be surprisingly simple, at least to a reasonable approximation. Silberberg [2] has argued that surfaces act like reflecting boundaries. One implication is that the distribution of any particular monomer, such as an end monomer, maintains its uniform bulk density all the way to the surface. Another is that the surface tension has no $N$ dependence.

Not surprisingly, the Silberberg argument is not exact. Indeed, measurements of the surface tension have detected a molecular-weight dependence [3-5]. Although experiments have yet to measure the distribution of chain ends, simulations [6-12] observe an excess of ends at the

\footnotetext{
a e-mail: mwmatsen@uwaterloo.ca
}

surface followed by a compensating depletion extending further into the melt. It remains to understand the reason for these violations of the Silberberg argument.

$\mathrm{Wu}$ et al. [13] showed that one source is the finite width, $\xi$, over which the surface profile, $\phi(z)$, drops to zero. They performed a self-consistent field theory (SCFT) calculation with a quadratic penalty for deviations from bulk density inversely proportional to the compressibility, $\kappa$, of the polymer melt. As they increased $\kappa$, the width of their surface profile increased as $\xi \propto \sqrt{\kappa}$, and with it emerged deviations from the Silberberg argument. In particular, they predicted an excess of chain ends at the surface followed by a compensating depletion that extended into the melt a distance proportional to $a N^{1 / 2}$. Furthermore, they predicted a reduction in the surface tension proportional to $N^{-1}$, which was reasonably consistent with the experimental measurements.

Simulations [12] have shown that the discreteness of a polymer chain also affects the behavior. This was demonstrated more explicitly by us [14] with a SCFT calculation similar to that of Wu et al., but for an incompressible melt of discrete chains. The calculation substituted the continuous Gaussian chain model used by $\mathrm{Wu}$ et al. with a model consisting of $N$ beads connected by freely-jointed bonds of arbitrary potential and solved the self-consistent field for a step profile (i.e., $\xi=0$ ). An analogous segregation of 
ends to the surface and a reduction in the surface tension were predicted, clearly illustrating that compressiblity is not the only source of deviation from the Silberberg argument. This begs the question as to what are the various sources and which of them are most important.

Here, we begin to answer this question by extending our earlier work to compressible melts, so as to combine the effects of chain discreteness with a finite width of the surface profile. Our initial intention was to include compressibility with the same quadratic energy term used by $\mathrm{Wu}$ et al., but this proved to be inadequate. After contemplating the issue, we decided that the more logical approach is to simply assume a reasonable functional form for the surface profile with an adjustable width, $\xi$, which in principle could be provided by a more advanced theory or by simulation. In this initial study, calculations are performed for two simple profile shapes, specifically sigmoidal and linear functional forms. Furthermore, a semianalytical approximation to the SCFT is developed to enhance our understanding and to ascertain what aspects are dependent upon microscopic details and which are universal.

\section{SCFT for discrete chains}

The system of this study is a neat melt of $n$ monodisperse polymers, each modeled as $N$ discrete monomers connected by freely-jointed bonds of some arbitrary potential. Each monomer is assigned a fixed volume, $\rho_{0}^{-1}$, such that the total volume of the melt is $V=n N / \rho_{0}$. A flat surface is imposed at $z=0$ from which the melt extends in the positive $z$ direction with a cross-sectional area of $\mathcal{A}$ in the $x-y$ plane.

The self-consistent field theory (SCFT) for this system is similar to that of our earlier study [14], and therefore we just provide a brief summary of the method mentioning the modifications and a couple computational improvements. As usual in SCFT, the non-bonded interactions are represented by an effective field, $w(z)$, that only depends on the coordinate, $z$, normal to the surface. For a discrete chain, the concentration of the $i$ 'th monomer relative to the bulk is given by

$$
\phi_{i}(z)=\frac{V}{\mathcal{Q}} \frac{G_{i}(z) G_{N+1-i}(z)}{h(z)},
$$

where $h(z) \equiv \exp \left(-w(z) / k_{B} T\right), G_{i}(z)$ is the partition function for a chain fragment of $i$ monomers with one end fixed at $z$, and

$$
\mathcal{Q} \equiv \mathcal{A} \int \frac{G_{i}(z) G_{N+1-i}(z)}{h(z)} d z
$$

is the single-chain partition function. Note that the normalization factor, $V / \mathcal{Q}$, in eq. (1) is such that $\phi_{i}(z) \rightarrow 1$ as $z \rightarrow \infty$. The propagator is evaluated using the recursive equation

$$
G_{i+1}(z)=h(z) \int g(Z) G_{i}(z-Z) d Z,
$$

starting from $G_{1}(z)=h(z)$. Our previous study [14] already considered the effect of different bonding potentials, and so this time we limit our attention to Hookean (or harmonic) bonds, for which

$$
g(Z)=\left(\frac{3}{2 \pi a^{2}}\right)^{1 / 2} \exp \left(-\frac{3 Z^{2}}{2 a^{2}}\right) .
$$

The field, $w(z)$, is adjusted so that the total polymer concentration,

$$
\phi(z)=\frac{1}{N} \sum_{i=1}^{N} \phi_{i}(z),
$$

equals some specified profile corresponding to a surface of width $\xi$ at $z=0$. Our study concentrates on the sigmoidal profile,

$$
\phi(z)=\frac{1}{2}\left[1+\tanh \left(\frac{2 z}{\xi}\right)\right],
$$

characteristic of detailed atomic simulations [11]. In order to gauge the sensitivity of our results to the actual shape of the profile, we repeat some of our calculations for a simple linear profile,

$$
\phi(z)= \begin{cases}0, & \text { if } z<-\frac{\xi}{2}, \\ \frac{1}{2}+\frac{z}{\xi}, & \text { if }-\frac{\xi}{2}<z<\frac{\xi}{2} \\ 1, & \text { if } \frac{\xi}{2}<z\end{cases}
$$

In our previous study [14], the self-consistent field, $w(z)$, was obtained using a simple mixing scheme, but this becomes inefficient as $\xi$ increases. To remedy the problem, we adopt an Anderson mixing algorithm $[15,16]^{1}$.

As usual, the SCFT is unaffected by an additive constant to the field, and so for convenience we define the field such that $w(z) \rightarrow 0$ as $z \rightarrow \infty$. With this choice, $G_{i}(z) \rightarrow 1$, which in turn requires the self-consistent solution to satisfy $\mathcal{Q}=V$ in order that $\phi(z) \rightarrow 1$ in the bulk. As a result, the dimensionless concentration of the end monomer simplifies to

$$
\phi_{e}(z) \equiv \phi_{1}(z)=G_{N}(z) \text {. }
$$

Furthermore, the surface tension is given by the simple expression

$$
\gamma_{\mathrm{en}}=-\rho_{0} \int w(z) \phi(z) d z
$$

Because our calculation creates the surface by constraining the polymer concentration instead of using molecular interactions (e.g., the quadratic treatment of compressibility), it just provides the entropic contribution to the surface tension due to chain connectivity. However, this is presumably the part responsible for the molecular-weight dependence. To obtain the complete surface tension, we would have to add the enthalpic contribution resulting from the molecular interactions.

1 The algorithm is the same as that used in ref. [16], except that the deviation at the $j$ 'th grid point of the $k$ 'th iteration is given by $d_{j}^{(k)}=\phi_{j}-\tilde{\phi}_{j}$, where $\phi_{j}$ is the actual concentration, eq. (5), and $\tilde{\phi}_{j}$ is the target concentration, eq. (6) or (7). 

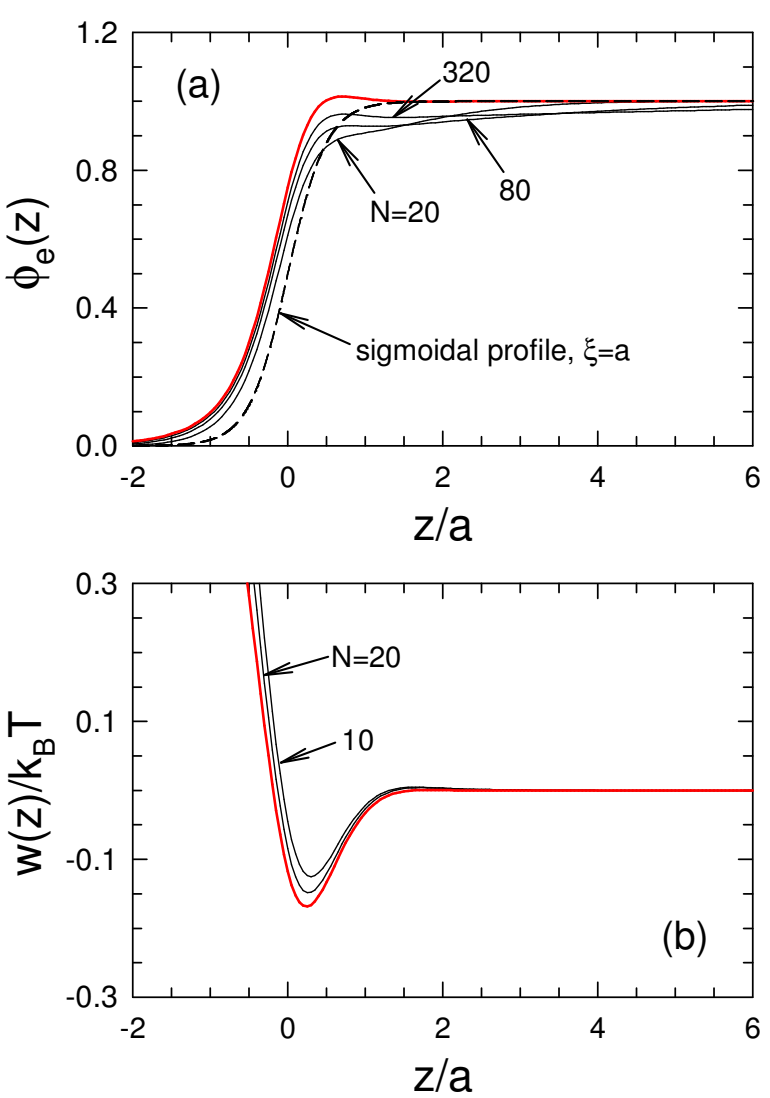

Fig. 1. (a) End-monomer distribution, $\phi_{e}(z)$, and (b) selfconsistent field, $w(z)$, calculated for a sigmoidal polymer profile, eq. (6), of width $\xi=a$, denoted by the dashed curve in plot (a). The black curves show results for different finite chain lengths, $N$, while the red curves denote the $N \rightarrow \infty$ limits.

Although the SCFT becomes increasingly computational for large $N$, the infinite molecular-weight limit is relatively simple to evaluate. This is partly because the polymer concentration reduces to

$$
\phi(z)=\frac{V}{\mathcal{Q}} \frac{G_{\infty}^{2}(z)}{h_{\infty}(z)},
$$

where the single-chain partition function is

$$
\mathcal{Q}=\mathcal{A} \int \frac{G_{\infty}^{2}(z)}{h_{\infty}(z)} d z
$$

In our previous study, we obtained the propagator for $N \rightarrow \infty$ by iterating eq. (3) several thousand times. A better approach, however, is to solve the integral equation,

$$
G_{\infty}(z)=\int I\left(z, z^{\prime}\right) G_{\infty}\left(z^{\prime}\right) d z^{\prime}
$$

where $I\left(z, z^{\prime}\right)=h_{\infty}(z) g\left(z-z^{\prime}\right)$. Discretization of the $z$ coordinate transforms this into a matrix equation, for which $G_{\infty}(z)$ is the eigenvector of the matrix $I\left(z, z^{\prime}\right)$ corresponding to the largest eigenvalue one. As such, $G_{\infty}(z)$ can be readily obtained from a standard linear algebra subroutine.
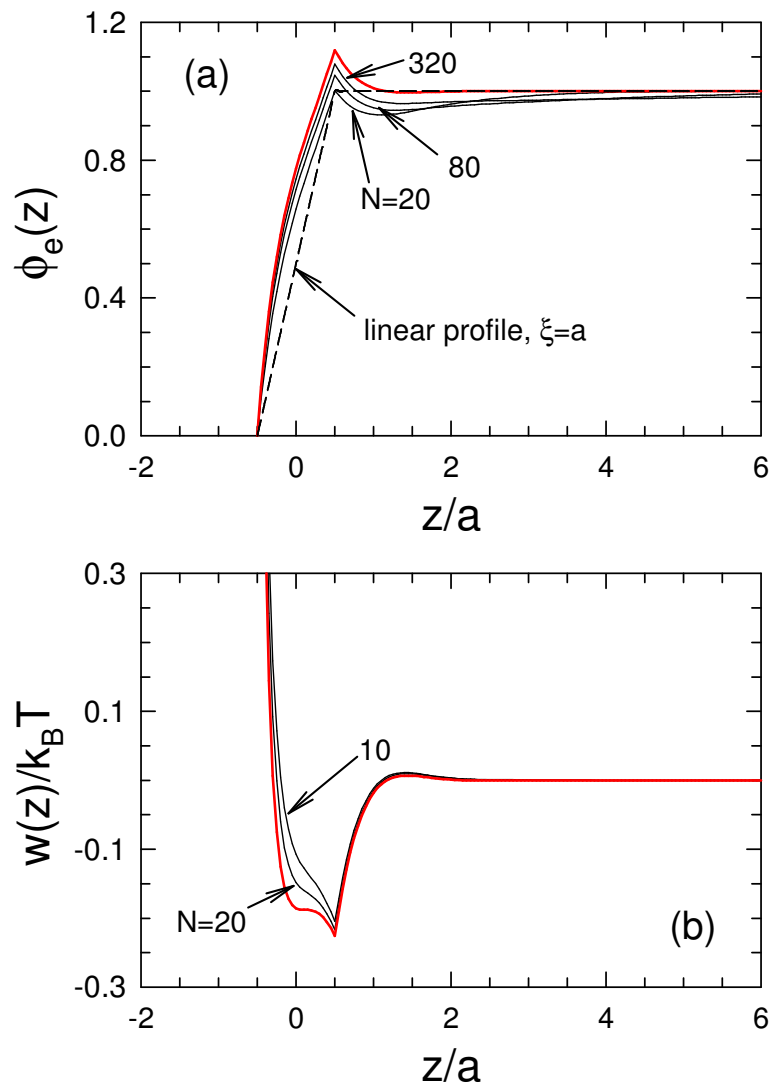

Fig. 2. Analogous plots to those of fig. 1, but calculated for a linear polymer profile, eq. (7), of width $\xi=a$.

\section{Results}

The width of a polymer surface is generally similar to the monomer size $[6,11,12]$, and so we begin by considering a sigmoidal profile with $\xi=a$. Figure 1a compares the end-monomer distribution, $\phi_{e}(z)$, for chains of finite $N$ (black curves) and infinite $N$ (red curve) to the overall polymer concentration, $\phi(z)$ (dashed curve). In all cases, there is an excess of ends near the surface (i.e., $\phi_{e}(z)>$ $\phi(z)$ for $z \lesssim a)$, which saturates as $N \rightarrow \infty$. The excess is then followed by a depletion, which becomes shallower and extends further into the melt as $N$ increases. Figure $1 \mathrm{~b}$ shows the field, $w(z)$, required to produce the sigmoidal profile. In this case, the effect of $N$ is so small that $w(z)$ becomes almost indistinguishable from the large- $N$ limit, $w_{\infty}(z)$ (red curve), once $N \gtrsim 100$. Analogous results are shown in fig. 2 for a linear profile, eq. (7), of width $\xi=a$. Although there are clear quantitative differences relative to those of the sigmoidal profile, the magnitude of the effects and the overall qualitative behavior is the same.

The total excess of ends at the surface is given by the integral

$$
\Delta \sigma_{e}=\frac{2 \rho_{0}}{N} \int_{-\infty}^{z_{0}}\left[\phi_{e}(z)-\phi(z)\right] d z
$$

where $z_{0}$ is the point at which $\phi_{e}\left(z_{0}\right)=\phi\left(z_{0}\right)$. This excess is then balanced by a depletion that extends into 


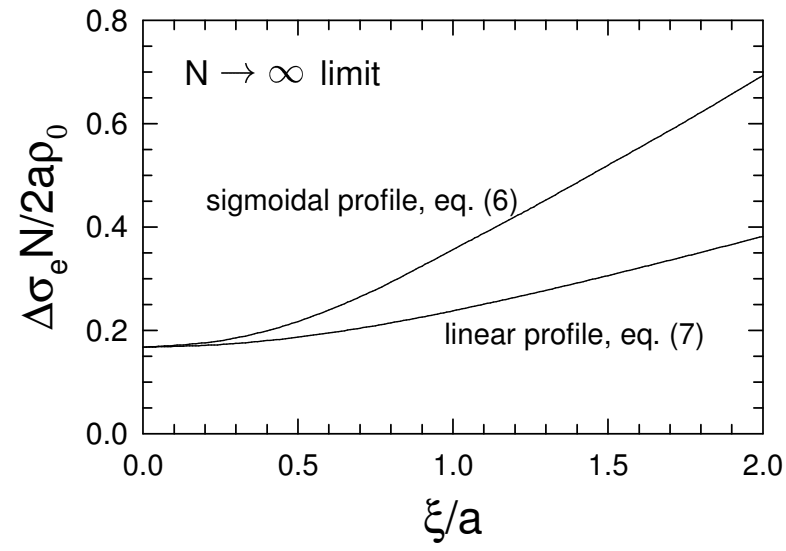

Fig. 3. Surface excess of ends per unit area, $\Delta \sigma_{e}$, plotted as a function of the width, $\xi$, of the concentration profile in the infinite molecular-weight limit. The dimensionless ratio of the vertical axis is precisely the $A$ appearing in eqs. (15), (16), (19) and (23).

the bulk. The range of the depletion is proportional to $N^{1 / 2}$ and thus the depth of the depletion scales as $N^{-1 / 2}$. Consequently, in the limit of $N \rightarrow \infty$, the end-monomer distribution, $G_{\infty}(z)$, contains an excess but no depletion. Hence, the excess for large $N$ can be approximated by

$$
\lim _{N \rightarrow \infty} \frac{\Delta \sigma_{e} N}{2 a \rho_{0}}=\frac{1}{a} \int_{-\infty}^{\infty}\left[G_{\infty}(z)-\phi(z)\right] d z
$$

which is plotted in fig. 3. From the plot, it is evident that the width of the profile, $\xi$, contributes to the excess by a comparable amount as the discreteness of the chain, which is responsible for the finite value at $\xi=0$.

In order to gain a deeper understanding of the behavior, we develop a semi-analytical approximation for $\phi_{e}(z)$ in terms of $G_{\infty}(z)$ (e.g., the red curves in figs. 1a and 2a). The calculation follows the same strategy used by refs. [13] and [14]. The first step is to solve the statistical mechanics of polymers of finite $N$ in the self-consistent field of infinitely long polymers, $w_{\infty}(z)$ (e.g., the red curves in figs. $1 \mathrm{~b}$ and $2 \mathrm{~b}$ ). We expect the propagator to take the form [14]

$$
\begin{aligned}
G_{N}^{0}(z) \approx & G_{\infty}(z)+A \sqrt{\frac{6}{\pi N}} \times \\
& \left\{\left[\exp \left(-\frac{3 z^{2}}{2 a^{2} N}\right)-1\right] H(z)+G_{\infty}(z)\right\}
\end{aligned}
$$

where $A$ is a constant that needs to be determined and $H(z)$ is the Heaviside function that switches from zero to one at $z=0$. This expression is such that the $N^{-1 / 2}$ correction is proportional to $G_{\infty}(z)$ for $z \lesssim a$ and proportional to a Gaussian for $z \gg a$. Given the propagator, we can now estimate the corresponding concentration, $\phi^{0}(z)$. This is done by inserting eq. (15) into eq. (1), and approximating the sum by an integral. Keeping terms to order $N^{-1 / 2}$, we obtain

$$
\phi^{0}(z) \approx \phi(z)+4 A \sqrt{\frac{6}{\pi N}} \frac{\phi(z)}{G_{\infty}(z)} \times
$$
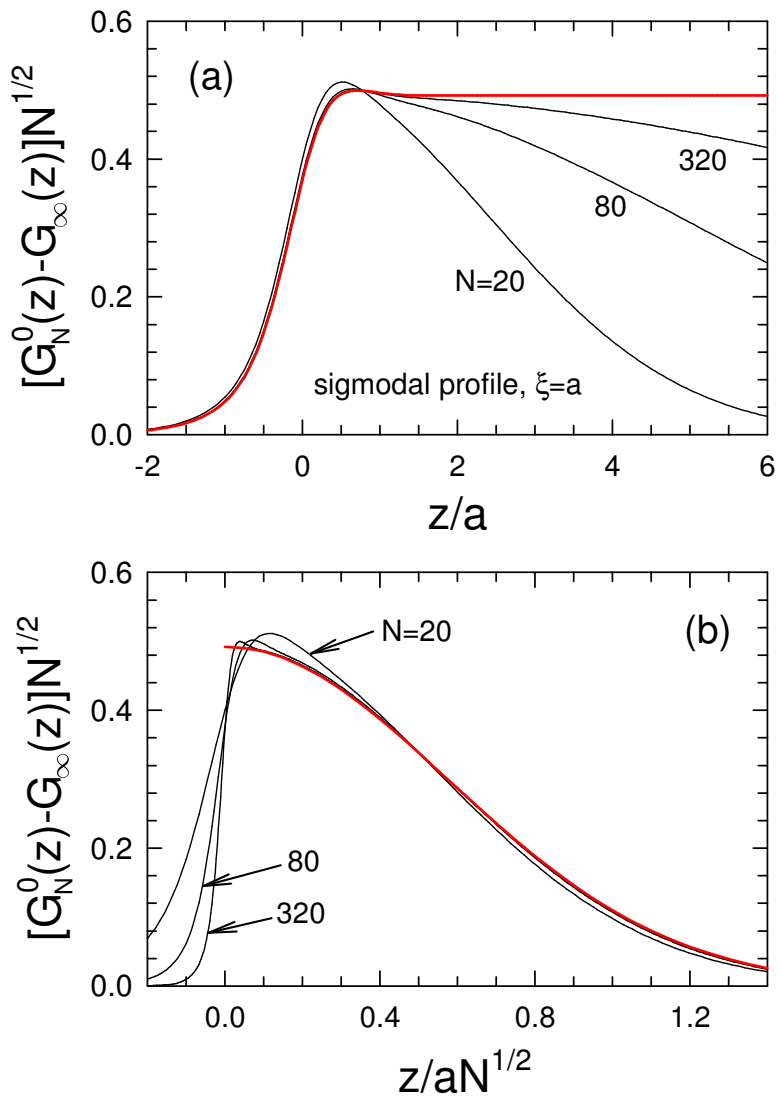

Fig. 4. Correction to $G_{N}^{0}(z) \approx G_{\infty}(z)$ plotted on the (a) monomer and (b) molecular length scales for different polymerizations, $N$. The red curves denote $A \sqrt{6 / \pi} G_{\infty}(z)$ and $A \sqrt{6 / \pi} \exp \left(-3 z^{2} / 2 a^{2} N\right)$, respectively.

$$
\left\{\left[f\left(\frac{z}{a N^{1 / 2}}\right)-1\right] H(z)+G_{\infty}(z)\right\}
$$

where

$$
f(\zeta)=\int_{0}^{1} \frac{1}{2 \sqrt{s}} \exp \left(-\frac{3}{2 s} \zeta^{2}\right) d s .
$$

The coefficient, $A$, in eqs. (15) and (16) is determined by examining the excess concentration of monomers to zeroth order in $N$. The excess of ends, which is given by the integral of $G_{N}^{0}(z)-\phi(z)$, has a short-range contribution equal to the integral of $G_{\infty}(z)-\phi(z)$ and a long-range contribution of $A a$. On the other hand, the excess of middle monomers, which is the integral of $\phi^{0}(z)-\phi(z)$, just has a long-range contribution of $2 A a$. The connectivity of the chains requires these excesses to balance, which implies that

$$
A=\frac{1}{a} \int_{-\infty}^{\infty}\left[G_{\infty}(z)-\phi(z)\right] d z .
$$

The validity of the above expressions, eqs. (15)-(18), is readily confirmed by performing numerical calculations for finite chains in the field $w_{\infty}(z)$. The red curves in figs. $4 \mathrm{a}$ and $\mathrm{b}$ show the short- and long-range corrections to $G_{N}^{0}(z)-G_{\infty}(z)$ predicted by eq. (15) for a sigmoidal profile of width $\xi=a$. The fact that the numerical results (black curves) converge to these limits as $N$ increases assures us 

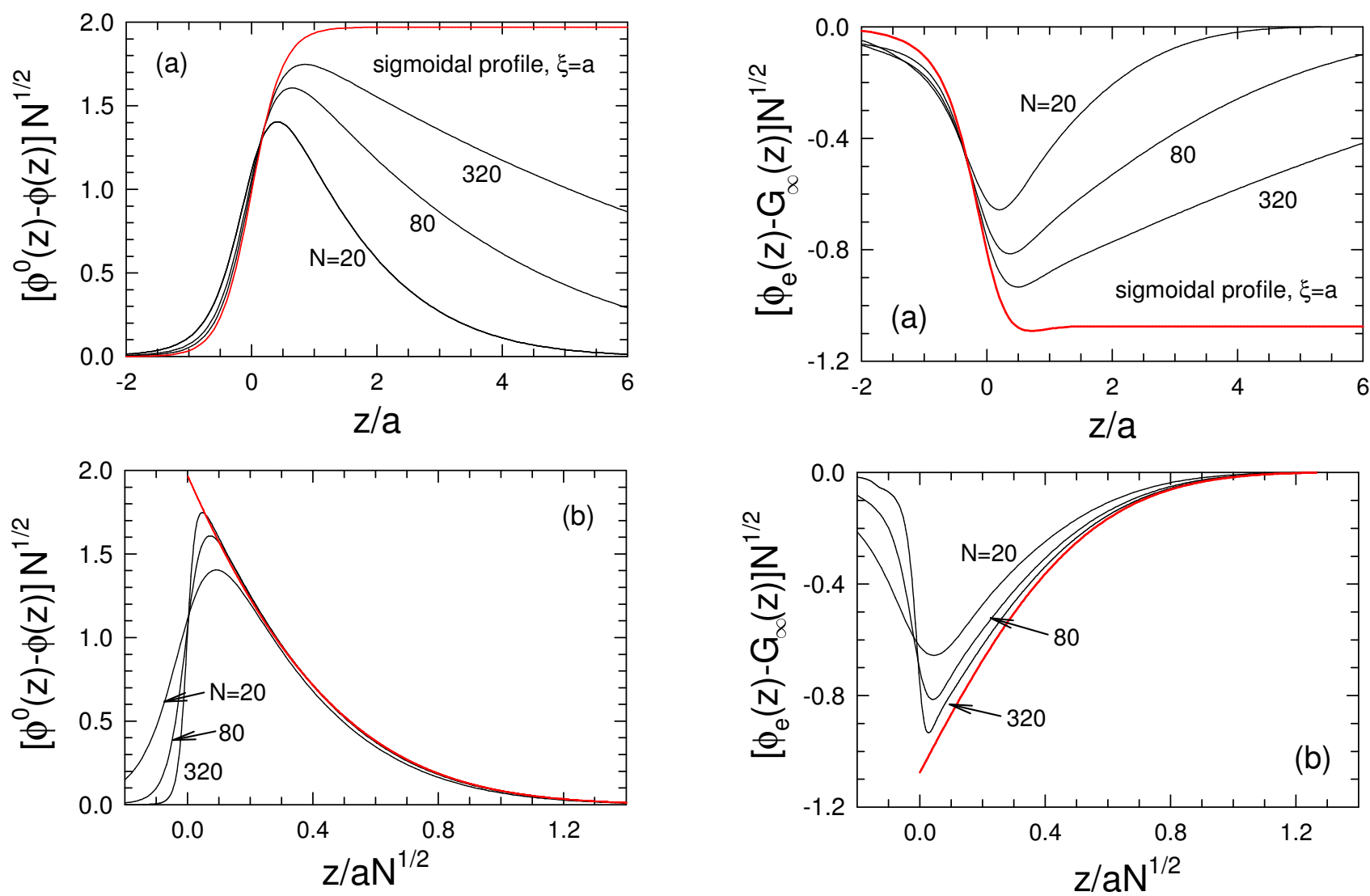

Fig. 5. Correction to $\phi^{0}(z) \approx \phi(z)$ plotted on the (a) monomer and (b) molecular length scales for different polymerizations, $N$. The red curves denote $4 A \sqrt{6 / \pi} \phi(z)$ and $4 A \sqrt{6 / \pi} f\left(z / a N^{1 / 2}\right)$, respectively.

that eq. (15) is correct. Similarly, the red curves in figs. 5a and $\mathrm{b}$ show the short- and long-range parts of the excess polymer concentration, $\phi^{0}(z)-\phi(z)$, predicted by eq. (16). Again the full numerical calculations for finite $N$ (black curves) converge to the expected limits (red curves).

The next step in the semi-analytical calculation is to adjust the field so as to remove the excess polymer concentration, $\phi^{0}(z)-\phi(z)$, plotted in fig. 5. Since the excess is small in amplitude and long range in extent, it can be corrected by using the linear response theory for continuous Gaussian chains [13]. More specifically, we calculate the long-range change in the field, $\delta w(z)$, required to remove the long-range excess concentration, eq. (17), denoted by the red curve in fig. 5b. This part of the semi-analytical calculation is exactly the same as in ref. [14].

The final step is to determine the change in $\phi_{e}(z)$ due to $\delta w(z)$, and this too can be done analytically using the linear response theory exactly as in ref. [14]. Adding the resulting correction to that of eq. (15) gives the final result,

$$
\begin{aligned}
& \phi_{e}(z) \approx G_{\infty}(z)+\frac{A}{N^{1 / 2}} \times \\
& \quad\left\{\left[B\left(\frac{z}{a N^{1 / 2}}\right)-B(0)\right] H(z)+B(0) G_{\infty}(z)\right\},
\end{aligned}
$$

Fig. 6. Correction to $\phi_{e}(z) \approx G_{\infty}(z)$ plotted on the (a) monomer and (b) molecular length scales for polymers of various polymerizations $N$, calculated for a sigmoidal surface profile of width $\xi=a$. The red curves denote $A B(0) G_{\infty}(z)$ and $A B\left(z / a N^{1 / 2}\right)$, respectively.

where

$$
B(\zeta)=\sqrt{\frac{6}{\pi}} \exp \left(-\frac{3}{2} \zeta^{2}\right)+b(\zeta)
$$

is a universal function. The $b(\zeta)$ is obtained ${ }^{2}$ by performing a numerical inverse Fourier transform of

$$
\hat{b}\left(k_{\zeta}\right)=-\sqrt{\frac{2}{\pi}} \frac{\left[\exp \left(-\frac{1}{6} k_{\zeta}^{2}\right)-1\right]^{2}}{\exp \left(-\frac{1}{6} k_{\zeta}^{2}\right)+\frac{1}{6} k_{\zeta}^{2}-1} .
$$

Figures $6 \mathrm{a}$ and $\mathrm{b}$ confirm the short- and long-range parts of the $N^{-1 / 2}$ correction in eq. (19) for a sigmoidal surface profile of width $\xi=a$. Likewise, the analogous plots in fig. 7 demonstrate the validity of the correction for a linear profile of width $\xi=a$.

Lastly, we evaluate the entropic contribution to the surface tension, $\gamma_{\mathrm{en}}$, using the expression in eq. (9). Figure 8 shows the tension in the large- $N$ limit,

$$
\Gamma_{\infty} \equiv \lim _{N \rightarrow \infty} \frac{\gamma_{\mathrm{en}}}{a \rho_{0} k_{B} T},
$$

\footnotetext{
2 The linear response theory is performed with a reflecting boundary condition at the surface, which treats $b(\zeta)$ as an even function
} 

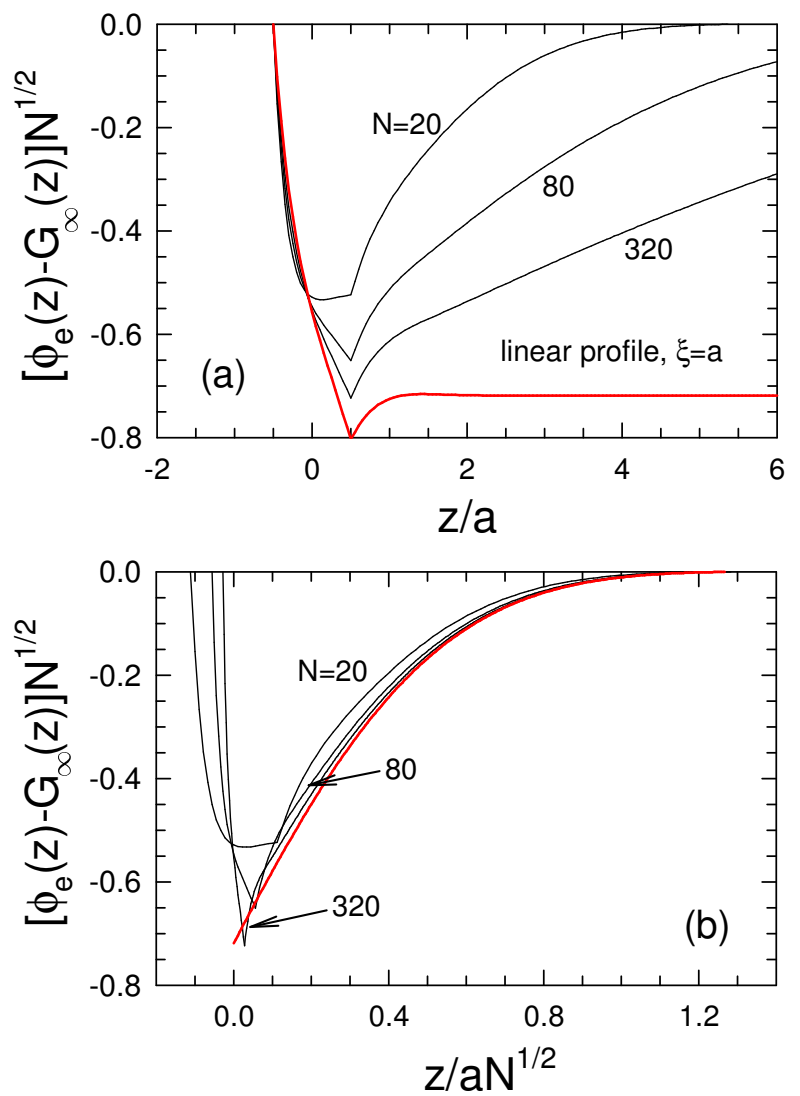

Fig. 7. Analogous plots to those in fig. 6, but calculated for a linear surface profile of width $\xi=a$.

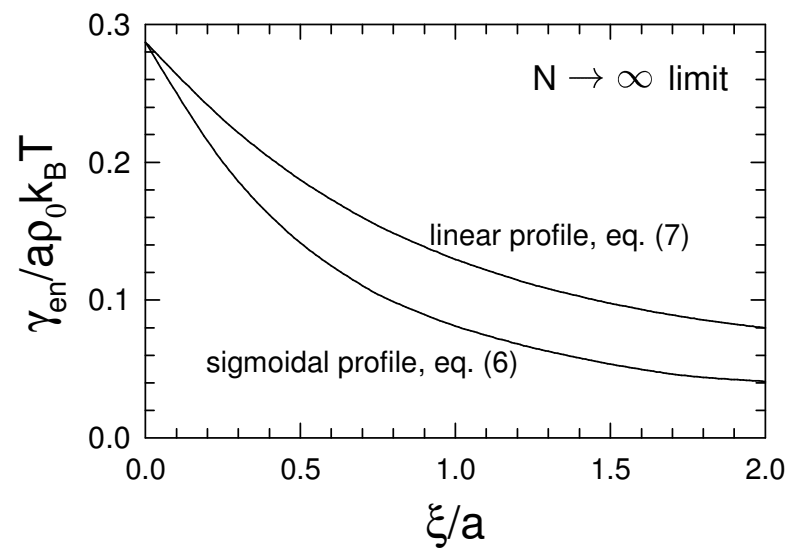

Fig. 8. Entropic contribution to the surface tension, $\gamma_{\mathrm{en}}$, as a function of the width, $\xi$, of the concentration profile in the infinite molecular-weight limit. The dimensionless ratio plotted on the vertical axis equates to the $\Gamma_{\infty}$ appearing in eq. (23).

$$
\begin{aligned}
& =-\frac{1}{a k_{B} T} \int_{-\infty}^{\infty} w_{\infty}(z) \phi(z) d z \\
& =\frac{1}{a} \int_{-\infty}^{\infty} \phi(z) \ln \left[\frac{G_{\infty}^{2}(z)}{\phi(z)}\right] d z
\end{aligned}
$$

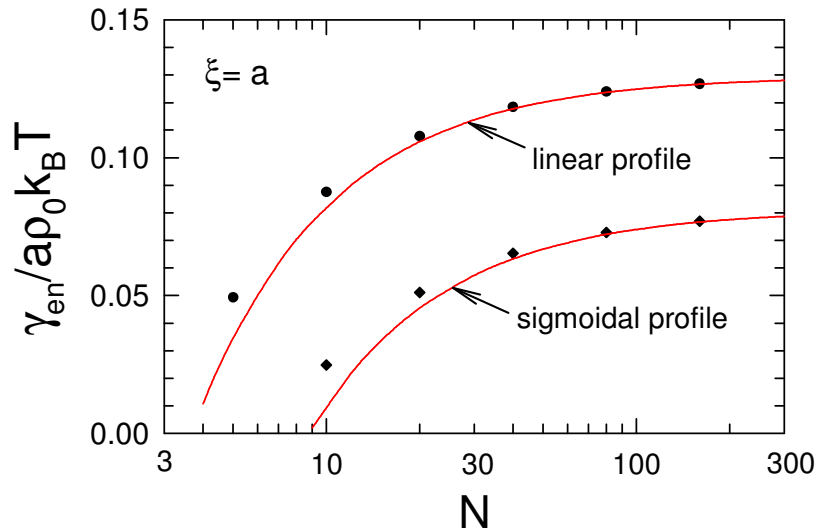

Fig. 9. Entropic contribution to the surface tension, $\gamma_{\mathrm{en}}$, as a function of polymerization, $N$. The symbols denote the full SCFT calculation while the red curves denote the approximation in eq. (23).
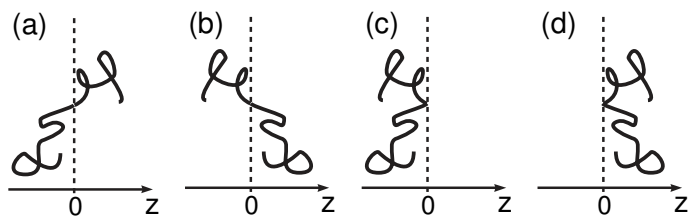

Fig. 10. Illustration of the Silberberg argument. (a) shows a polymer taken from the bulk, while (b)-(d) show configurations of equivalent energy (and thus probability) obtained by reflections about the plane $z=0$. The surface is created by removing (a)-(c) and doubling the probability of (d).

as a function of the width $\xi$ of the surface profile. For finite $N$, we can use the approximation derived in ref. [14],

$$
\frac{\gamma_{\mathrm{en}}}{a \rho_{0} k_{B} T} \approx \Gamma_{\infty}-\frac{2 A}{N}
$$

where $A$ is now the coefficient from eq. (18) plotted in fig. 3. Figure 9 illustrates the accuracy of this approximation (red curves) by comparing to the full SCFT calculation (symbols). As we can see, it remains reasonably accurate for polymer chains as short as ten or twenty monomers.

\section{Discussion}

As mentioned in the Introduction, the Silberberg argument [2] implies that there should be no excess of chain ends at the surface and no $N$-dependence in the surface tension. To understand how this can nevertheless happen, it is necessary to consider the actual argument and the assumptions behind it. The argument starts by considering an infinite polymer melt and then removing all the material at $z<0$. Figure 10a shows a polymer configuration that crosses $z=0$, dividing the molecule into two portions; the subsequent configurations, b, c and d, are obtained by reflecting one or both of these portions about $z=0$. Assuming the molecule is flexible, all four configurations will have the exact same energy and therefore will occur in 
the bulk with equal probability. To create the surface, the configurations in figs. 10a, b and c must be removed. Naturally, the absence of a and b will reduce the polymer concentration at $z>0$, but this can be exactly compensated for by doubling the concentration of the configuration in fig. 10d. In general, if a polymer crosses the surface $m$ times, the weight of the allowed configuration, obtained by reflecting the portions of the molecule at $z<0$, has to be increased by a factor of $2^{m}$. This is achieved by applying a Dirac delta potential at $z=0$. Not only does the procedure maintain the uniform polymer density for $z>0$, it does so for all the individual monomers including the ends. Furthermore, the free energy cost of creating the surface is just proportional to the number of bonds that initially crossed the $z=0$ plane, which is independent of how long the molecules are.

Notice that this procedure only applies to a step profile (i.e., $\xi=0$ ), which is why $\mathrm{Wu}$ et al. [13] observed violations to the Silberberg argument for compressible melts. Also notice that the argument assumes that reflecting the polymer about an arbitrary plane does not alter its energy. Although this is true for continuous Gaussian chains, it is not true for discrete chains because the reflection shortens the bond that initially straddles the plane [17]. This explains why our previous study [14] also found violations to the argument even though it was for an incompressible melt. Now that the assumptions are clear, we can anticipate other things that would affect the segregation of chain ends. An obvious one would be chain stiffness [19]. Another would be the explicit inclusion of hard-core interactions between monomers, given that reflections can cause a chain to overlap itself $[20,21]$.

When contemplating how to combine the effects of compressibility and chain discreteness, our initial thought was to apply the quadratic treatment of compressibility from ref. [13] to the bead-spring model from ref. [14], but this lead to unphysical behavior. The problem is that the simple quadratic treatment causes the melt to fill all the available space, just like a gas. This is evident by the fact that a wall is required in order to create a surface. While this results in a reasonable profile for Gaussian chains in that $\phi(z)$ drops continuously to zero $[17,18]$, it produces a discontinuous profile for discrete chains [12]. We considered using a more sophisticated treatment combining SCFT with density functional theory [23,24], but it made more sense to simply impose the shape of the profile. The added complexity of incorporating appropriate interactions would just have acted to obscure the physics that we are interested in.

We should point out that just because the quadratic treatment of compressibility results in a smooth profile for Gaussian chains does not imply that the surface behaves properly. For instance, there is no reason to trust its prediction that $\xi$ decreases for small $N[12,13]$. Indeed, microscopic simulations based on Lennard-Jones interactions have predicted either a negligible molecular-weight dependence [7] or a slight increase [11]. Since $\xi$ is simply an input parameter in our calculation, we can readily incorporate an $N$ dependence. It just implies that, for example, the $A$ and $\Gamma_{\infty}$ coefficients in eq. (23) for the surface tension would have an implicit $N$-dependence though their explicit dependence on $\xi$ in figs. 3 and 8 , respectively.

An advantage of assuming a profile shape, rather than creating it with molecular interactions, is the resulting tractability of our semi-analytical calculation. The analogous calculation by $\mathrm{Wu}$ et al. [13] with the quadratic treatment of compressibility lead to a complicated expression for their version of $G_{\infty}(z)$. As a result, they had to perform the last part of the calculation numerically. In contrast, we begin with a simple numerical evaluation of $G_{\infty}(z)$ and then the rest of the calculation proceeds analytically. One benefit of our approach is the analytical prediction for the long-range depletion, $B\left(z / a N^{1 / 2}\right)$. Wu et al. would have obtained the same expression had they been able to complete the latter part of their calculation analytically.

Another universal prediction from our semi-analytical calculation is that the reduction in $\gamma_{e n}$ is proportional to $A$, which in turn is proportional to $\Delta \sigma_{e}$. As mentioned earlier, only this entropic part of the surface tension should have a molecular-weight dependence; the other contributions will just involve details related to the surface region $(0<z \lesssim \xi)$ and as such become independent of $N$ for large polymers. Consequently, the total surface tension should obey

$$
\gamma \approx \gamma_{\infty}-k_{B} T \Delta \sigma_{e}
$$

where $\gamma_{\infty}$ is the infinite molecular-weight limit. Note that this is similar to a result hypothesized by Kumar et al. [8]. The semi-analytical calculation now provides theoretical justification for it.

Part of our reason for considering two different surface profiles, eqs. (6) and (7), was to distinguish between model-dependent and universal behavior. The propagator for infinite chains, $G_{\infty}(z)$, and the resulting coefficients, $A$ and $\Gamma_{\infty}$, calculated from eqs. (14) and (22), respectively, are clearly dependent upon the details of the model. This tells us that a quantitative calculation of the surface excess of chain ends (i.e., $A$ ) would require an atomically realistic model, and is thus beyond the scope of a coarsegrained approach. Nevertheless, we can trust the qualitative trends, such as the general increase in $\phi_{e}(z)$ at the surface as $N \rightarrow \infty$, which is indeed consistent with simulations $[7,8,10]$. On the other hand, the universal predictions are expected to be quantitatively accurate for all models provided $N$ is sufficiently large. This includes the shape of the of the compensating depletion in eq. (20) and the effect $\Delta \sigma_{e}$ has on the surface tension in eq. (24).

The cleanest way to test our predictions would be by computer simulation, which typically uses off-lattice beadspring models much like that of our calculation. In fact, numerous studies $[6-12]$ have already detected the segregation of ends to the surface, and furthermore Müller et al. [12] have explicitly observed the effect of chain discreteness. It should be reasonably straightforward to confirm our prediction for the compensating depletion of chain ends. Although surface tension is difficult to extract from simulation, it would be worthwhile to also validate the relationship in eq. (24). However, to test these predictions, 
the simulation would need to be performed at high molecular weights (e.g. $N \gtrsim 100$ ), where the molecular size, $a N^{1 / 2}$, is large relative to the monomer size, $a$, and the width of the surface, $\xi$.

Experiments would not be able to isolate entropic effects like those of our study. While simulations can ensure that end and middle monomers interact identically, this is not generally true of experiments $[25,26]$ particularly if the ends have to be labeled in order to measure $\phi_{e}(z)$. The difference in interaction will equate to an effective surface potential, $w_{e}(z)$, acting on the ends. Nevertheless, the modification to our calculation would be minimal. The chain-end concentration in eq. (8) would become $\phi_{e}(z)=h_{e}(z) G_{N}(z)$, where $h_{e}(z) \equiv \exp \left(-w_{e}(z) / k_{B} T\right)$. The propagator for an infinite chain, $G_{\infty}(z)$, would be unaffected, and eqs. (15) and (16) for $G_{N}^{0}(z)$ and $\phi^{0}(z)$ would continue to hold but with a modified coefficient of

$$
A=\frac{1}{a} \int_{-\infty}^{\infty}\left[h_{e}(z) G_{\infty}(z)-\phi(z)\right] d z .
$$

As such, the long-range depletion of ends will still have the same universal shape, $B\left(z / a N^{1 / 2}\right)$, and the correction to the surface tension should continue to obey eq. (24).

\section{Summary}

We have investigated the entropic segregation of chain ends to the surface of a polymer melt using self-consistent field theory (SCFT). The calculation assumes a surface profile of width $\xi$ and treats the polymers using a discrete bead-spring model with a polymerization of $N$ and a statistical bond length of $a$. The finite surface width and the discreteness of the chain are found to have a comparable effect on the surface excess of ends, $\Delta \sigma_{e}$, assuming $\xi \sim a$. The surface excess is then balanced by a long-range depletion that extends a molecular distance, $a N^{1 / 2}$, into the melt. The segregation of chain ends is also directly linked to a reduction in surface tension.

In addition to the SCFT calculation, we also developed a semi-analytical approximation that provides a deeper understanding of the effects. It begins with a simple numerical evaluation of the partition function, $G_{\infty}(z)$, of an infinite chain with one end fixed at $z$. From that, we derive analytical expressions for the end-monomer distribution, $\phi_{e}(z)$, and the entropic contribution to surface tension, $\gamma_{\mathrm{en}}$, for finite chains, eqs. (19) and (23), respectively. The coefficients in these expressions, $A$ and $\Gamma_{\infty}$, are evaluated from $G_{\infty}(z)$ using eqs. (14) and (22). The function $G_{\infty}(z)$ and the resulting coefficients are model dependent, which implies that a quantitative prediction of $\Delta \sigma_{e}$ would require a realistic model of the polymer. Nevertheless, we do find a general increase in the relative concentration of ends, $\phi_{e}(z)$, at the surface as $N \rightarrow \infty$, consistent with simulations $[7,8,10]$. Interestingly, the calculation predicts a universal shape, $B\left(z / a N^{1 / 2}\right)$, for the compensating depletion of chain ends, and a reduction in surface tension equal to $-k_{B} T \Delta \sigma_{e}$ irrespective of the model details.
The challenge now is to test our universal predictions for the compensating depletion and the reduction in surface tension. The most direct way would be by computer simulation, which generally uses very similar bead-spring models where all monomers are treated identically. In experiments, end monomers will interact somewhat differently than middle monomers. Nevertheless, the resulting enthaplic contribution to $\Delta \sigma_{e}$ should not alter the shape of the compensating depletion nor the reduction in surface energy per chain end.

This work was supported by start-up funds from the University of Waterloo.

\section{References}

1. J.P. Wittmer, A. Cavallo, H. Xu, J.E. Zabel, P. Poliüska, N. Schulmann, H. Meyer, J. Fatago, A. Johner, S.P. Obukhov, J. Baschnagel, J. Stat. Phys. 145, 1017 (2011).

2. A Silberberg, J. Colloid Interface Sci. 90, 86 (1982).

3. C. Jalbert, J.T. Koberstein, I. Yilgor, P. Gallagher, V. Krukonis, Macromolecules 26, 3069 (1993).

4. S.H. Anastasiadis, I. Gancarz, J.T. Koberstein, Macromolecules 21, 2980 (1988).

5. B.B. Sauer, G.T. Dee, J. Coll. Interf. Sci. 162, 25 (1994).

6. S.K. Kumar, M. Vacatello, D.Y. Yoon, J. Chem. Phys. 89, 5206 (1988).

7. I. Bitsanis, G. Hadziioannou, J. Chem. Phys. 92, 3827 (1990).

8. S.K. Kumar, M. Vacatello, D.Y. Yoon, Macromolecules 23, 2189 (1990).

9. A. Yethiraj, C. K. Hall, Macromolecules 23, 1865 (1990).

10. R.S. Pai-Panandike, J.R. Dorgan, T. Pakula, Macromolecules 30, 6348 (1997).

11. K.C. Daoulas, V.A. Harmandaris, V.G. Mavrantzas, Macromolecules 38, 5780 (2005).

12. M. Müller, B. Steinmüller, K.C. Daoulas, A RamírezHernández, J.J. de Pablo, Phys. Chem. Chem. Phys. 13, 10491 (2011).

13. D.T. Wu, G.H. Fredrickson, J.-P. Carton, A. Ajdari, L. Leiber, J. Polym. Sci., Part B 33, 2373 (1995).

14. M.W. Matsen, P. Mahmoudi, Eur. Phys. J. E 37, 78 (2014).

15. D.G. Anderson, J. Assoc. Comput. Mach. 12, 547 (1965).

16. P. Stasiak, M.W. Matsen, Eur. Phys. J. E 34, 110 (2011).

17. M.W. Matsen, J.U. Kim, A.E. Likhtman, Eur. Phys. J. E 29, 107 (2009).

18. I.Y. Erukhimovich, A. Johner, J.F. Joanny, Eur. Phys. J. E 27, 435 (2008).

19. D.C. Morse, G.H. Fredrickson, Phys. Rev. Lett. 73, 3235 (1994).

20. M. Müller, J. Chem. Phys. 116, 9930 (2002).

21. A. Cavallo, M. Müller, J.P. Wittmer, A. Johner, K. Binder, J. Phys.: Conds. Matter 17, S1697 (2005).

22. C.E. Woodward, J. Chem. Phys. 97, 695 (1993).

23. S.K. Nath, J.D. McCoy, J.P. Donley, J.G. Curro, J. Chem. Phys. 103, 1635 (1995).

24. F. Schmid, J. Chem. Phys. 104, 9191 (1996).

25. P.G. de Gennes, C. R. Acad. Sci. Paris 307, 1841 (1988).

26. J.H. Jang, R. Ozisik, W.L. Mattice, Macromolecules 33 7663 (2000). 